\title{
Verbreitung und Akzeptanz von Bauchentscheidungen im Management
}

\author{
Barbara Krumay (D) David Rückel $\cdot$ Philipp Wicke $\cdot$ Stefan Koch
}

Eingegangen: 6. April 2018 / Angenommen: 24. Mai 2018 / Online publiziert: 7. Juni 2018

(C) Der/die Autor(en) 2018

Zusammenfassung Das Treffen von unternehmerischen Entscheidungen auf Basis großer Datenmengen, die mittels Informationssystemen strukturiert, analysiert und visualisiert werden, stellt seit Jahrzehnten ein Kernthema der Wirtschaftsinformatik dar. In diesem Fall spricht man von einer fakten- oder evidenzbasierten Entscheidung (System 2). Gleichzeitig werden in Unternehmen (bewusst) Entscheidungen ohne die Verwendung solcher Daten getroffen. In diesem Fall spricht man von einer intuitiven Entscheidung (System 1) oder auch der „Bauchentscheidung“. Intuitive Entscheidungen beruhen meist auf der Erfahrung der EntscheidungsträgerInnen und werden in der Regel schneller getroffen, da langwierige Datensammlung und -aufbereitung entfallen. Intuitive Entscheidungen werden oft kritisch betrachtet, da die faktenbasierte Grundlage fehlt. Diese intuitiven Entscheidungen wurden bisher in der Wirtschaftsinformatik nur am Rande betrachtet. Allerdings besteht in jenen Unternehmen, die langfristig das Wissen über getroffene Entscheidungen (also retrospektiv) dazu nutzen wollen, zukünftige Entscheidungen schneller zu treffen, der Bedarf, intuitive Entscheidungen mit Informationssystemen zu unterstützen. Der Bedarf bezieht sich sowohl auf den Prozess der Entscheidung als auch die Dokumentation, um aus ähnlichen Entscheidungssituationen lernen zu können, ob eine intuitive oder eine faktenbasierte Entscheidung zu bevorzugen ist. Dieser Artikel untersucht empirisch durch Interviews sowie einer Umfrage unter ManagerInnen die Akzeptanz von und das Bewusstsein für intuitive Entscheidungen. Daraus werden Erkenntnisse für den Einsatz von Informationssystemen zur Unterstützung von intuitiven Entscheidungen abgeleitet. Aus Sicht der Wissenschaft trägt dieser Artikel somit zu einer breiteren

\footnotetext{
B. Krumay $(\bowtie) \cdot$ D. Rückel $\cdot$ S. Koch Institut für Wirtschaftsinformatik - Information Engineering, Johannes Kepler Universität Linz, Linz, Österreich

E-Mail: barbara.krumay@jku.at

P. Wicke

TD Trusted Decisions GmbH, Bahnhofstraße 8, 30159 Hannover, Deutschland
} 
Diskussion im Bereich Decision Engineering und Wissensmanagement bei. Für die Praxis wird aufgezeigt, wie das Wissen um intuitive Entscheidungen genutzt werden kann, um in künftigen Entscheidungssituationen das adäquate Entscheidungssystem - rational oder intuitiv - anzuwenden.

Schlüsselwörter Entscheidungsunterstützung · Intuitive Entscheidungen ·

Rationale Entscheidungen · Decision Engineering · Bauchentscheidung

\section{Adoption and Acceptance of Gut Decisions in Management}

Abstract The support of managerial decisions through information systems structuring, analysing and visualising data is a common research topic in business informatics. While this is valid for fact based or rational decisions (System 2), decisionmakers frequently make intuitive decisions that are not based on evidence or facts. These decisions are also called System 1 decisions or "gut decisions". Such intuitive decisions are based on decision-makers' experience. On one hand, they are made faster, as data does not have to be collected and analysed. On the other hand, they are subject to being questioned due to their lack of evidence. Even though this topic has not yet been in the focus of business informatics research, there is a managerial demand for decision support for intuitive decision-making. A decision support system should cover both decision process and documentation to learn from prior decisions' outcomes. Therefore, qualitative and quantitative research has been performed to investigate the state of the art on intuitive decision making in companies. Furthermore, factors for the design of such a decision support system are derived.

Keywords Decision support - Intuitive decision making $\cdot$ Rational decision making $\cdot$ Decision engineering

\section{Entscheidungen und Unternehmenserfolg}

Der Erfolg eines Unternehmens hängt nicht zuletzt davon ab, welche Entscheidungen ManagerInnen treffen. Diese Entscheidungen bestimmen, welche Produkte entwickelt werden, welche Personen eingestellt werden, oder wie Ressourcen eingesetzt werden. Auch wenn viele Bereiche - von der Wirtschaftsinformatik über das Wissensmanagement bis hin zur Psychologie - sich seit langem damit beschäftigen, wie Entscheidungen unterstützt werden können, bleibt das Treffen von Entscheidungen unmittelbar mit dem Faktor Mensch verbunden. Auch in der Wirtschaftsinformatik wird die Unterstützung von Entscheidungen mit Hilfe von Decision Support Systemen (DSS) seit langem thematisiert (Mertens 1999). Auch Ansätze des evidenzbasierten Managements stützen sich auf Daten als Basis für Entscheidungen (Pfeffer und Sutton 2006). Im Wissensmanagement wird sowohl das Bewahren von Wissen als Voraussetzung für darauf basierende Entscheidungen diskutiert (Alavi und Leidner 2001), als auch, wie Entscheidungen in wissensintensiven Prozessen unterstützt werden können (van Helden et al. 2018). Doch es zeigt sich, dass im betrieblichen Alltag Entscheidungen nicht rein rational getroffen werden, sondern dass die 
Intuition gerade vor dem Hintergrund der Digitalisierung noch von Bedeutung ist. Auch wenn Intuition im Management oft kritisch betrachtet wird, sind intuitive Entscheidungen unternehmerischer Alltag (Styhre 2011). Man unterscheidet zwischen „Bauchentscheidungen“, also dem intuitiven Entscheiden (System 1), basierend auf Erfahrung des Individuums und dem rationalen Entscheiden (System 2), basierend auf Fakten (Stanovich und West 2000). Die Unterstützung durch Informationssysteme fokussiert bisher stark auf rationale Entscheidungen, also letztendlich darauf, die Fakten aufzubereiten.

Obwohl IT-Unterstützung Individuen helfen könnte, Entscheidungen intuitiv oder rational auf Basis von existierendem Wissen und Erfahrung zu treffen, gibt es auf dem Gebiet bisher wenige Erkenntnisse. Ein Informationssystem kann vor allem den Prozess der Entscheidung unterstützen, indem es die entsprechenden Einflussfaktoren abbildet. Darüber hinaus können Informationssysteme zur Dokumentation von Entscheidungen herangezogen werden, um das Wissen zu bewahren oder in der Rückschau zu betrachten, inwiefern eine Entscheidung zum gewünschten Ergebnis geführt hat. Aufbauend darauf können auch zukünftige Entscheidungen (besser) getroffen werden. Der Diskurs, wie Informationssysteme in dieser Situation Individuen unterstützen können - überwiegend angesiedelt in den Bereichen Wirtschaftsinformatik, Decision Engineering und Wissensmanagement - steht noch am Anfang. Ziel dieser Arbeit ist es daher, Anforderungen an zukünftige Informationssysteme abzuleiten, die Individuen unterstützen sollen, bewusst zwischen intuitiven und rationalen Entscheidungen wählen zu können, diese Entscheidungen zu beurteilen und aus diesen Entscheidungssituationen für weitere, zukünftige Entscheidungen zu lernen. Die Forschungsfrage dieser Arbeit lautet demgemäß, welche Anforderungen an ein Informationssystem gestellt werden, das einerseits in der Entscheidungssituation den Menschen unterstützen kann, andererseits aber auch Entscheidungen dokumentieren kann, um zukünftig auf diese Erfahrung zurückgreifen zu können.

\section{Stand der Forschung zu intuitivem und rationalem Entscheiden}

Wie Entscheidungen am besten getroffen werden (Dane und Pratt 2007) ist seit Jahrzehnten in vielen Disziplinen (z. B. Philosophie, Psychologie, Wirtschaft) Gegenstand der Forschung. Obwohl eine endgültige Antwort auf diese Frage nicht existiert, hat die Wissenschaft Modelle entworfen, wie Entscheidungen getroffen werden sollen (normativ), und Möglichkeiten geschaffen, um getroffene Entscheidungen zu beschreiben (deskriptiv) (Meyer 2000). Generell spricht man von einer Entscheidung, wenn man zwischen Handlungsalternativen, die geeignet sind ein Ziel zu erreichen, wählen kann (Meyer 2000). Die Idee des Homo oeconomicus, einem Individuum mit vollständigem Wissen und unlimitierten Ressourcen, das in seiner Entscheidung auf maximalen Nutzen abzielt, spielt in der Ökonomie eine große Rolle (Gintis 2000). Entsprechend dem ökonomischen (rationalen) Prinzip, wird die Alternative gewählt, bei der unter Verwendung aller relevanten Informationen Input und Output in einer optimalen Beziehung stehen (Shapiro und Krishnan 2001). Diese idealisierte Situation, in der alle relevanten Informationen vorhanden sind, findet sich zwar in vielen bekannten Ansätzen, z. B. auch der Bayes'schen Ent- 
scheidungstheorie, ist allerdings nur für sogenannte kleine Welten gültig (Savage 1951).

Rationale Entscheidungen, die in der Betriebswirtschaft idealisiert werden (Day 1971), können die Komplexität von Entscheidungssituationen häufig nicht ausreichend abbilden (Lank und Lank 1995; Huang 2017). Auch existierende mathematische Modelle zur Unterstützung rationaler Entscheidungen sind nur in der Lage, Wahrscheinlichkeitsaussagen über die Zukunft zu treffen (Sayegh et al. 2004), da vollständiges Wissen nicht vorhanden ist und valide Aussagen über die Zukunft nicht gemacht werden können. Darüber hinaus sind diese Ansätze durch die dynamische Umgebung, in der sich Ausgangs- und Randbedingungen ständig ändern, für viele Entscheidungen nur bedingt geeignet (Goll und Rasheed 1997).

Issack (1978) hat schon früh gefordert, die Erforschung der Intuition im Management zu forcieren. Aber erst in letzter Zeit rücken intuitive Entscheidungen immer mehr in den Mittelpunkt des Forschungsinteresses (Lank und Lank 1995; Sadler-Smith und Shefy 2004; Huang 2017). Es scheint klar, dass Entscheidungen nicht strikt rational, sondern intuitiv (Kahneman und Frederick 2002; Sadler-Smith und Shefy 2004), basierend auf Erfahrungen aus vergleichbaren Situationen (Sitkin und Weingart 1995), getroffen werden. Dane und Pratt (2007) definieren Intuition als affektiv aufgeladene Entscheidungen, die durch schnelle, unbewusste und ganzheitliche Assoziationen entstehen. Simon (1987) erklärt Intuition am Beispiel eines Kindes, das einen Hund erkennt und sofort das Bellen imitiert.

In der Wissenschaft wird zwischen System 1-Denken (intuitiv) und System 2Denken (analytisch) unterschieden (Stanovich und West 2000; Kahneman und Frederick 2002). System 1-Denken beruht auf Verallgemeinerungen sowie Abstraktionen mit Hilfe von Prototypen, Skripten, Metaphern und Erzählungen (Stanovich und West 2000). Epstein (1994) verbindet System 1-Denken mit einem „Erfahrungsmodus“, der von Menschen aber auch Tieren verwendet wird. System 2-Denken ist unabhängiger von Gefühlen als System 1-Denken, stellt höhere Anforderungen an die kognitiven Fähigkeiten und entwickelt sich im Lauf der Zeit (Stanovich und West 2000). Es ist nicht nur analytisch, sondern auch kontrollierbar, regelbasiert, und bewusst. Obwohl zwischen den beiden Systemen ein strikter Unterschied besteht, wenden Individuen beide Systeme an (Stanovich und West 2000), da die Systeme nicht unabhängig voneinander existieren (Milkman et al. 2009). System 1-Denken kann sich in vielen Alltagssituationen wie ein Standardsystem verhalten. Wenn Individuen Muster der Entscheidungssituation verstehen oder wiederholt etwas tun, werden Entscheidungen automatisch ohne hohe kognitive Belastung getroffen. Wenn ein Problem oder eine Unregelmäßigkeit auftritt, wechseln Individuen automatisch zu System 2-Denken als Interventionsinstanz, die in System 1-Denken eingreifen, dieses jedoch nicht völlig ausschalten kann, was auch zu Konflikten zwischen den Systemen führen kann (Stanovich und West 2000). Wenn ManagerInnen „eine positivere Haltung gegenüber ihrer eigenen intuitiven Fähigkeit einnehmen“" (Agor 1986) und ihre Intuition für strategische Entscheidungen nutzen, lernen sie gleichzeitig daraus. Gute Ergebnisse scheinen vor allem durch die Kombination intuitiver und rationaler Entscheidungsprozesse erzielt zu werden (Milkman et al. 2009). 


\section{Methode und Ergebnisse}

Zur Erreichung des Forschungsziels wurde eine zweistufige, empirisch-behavioristische Methodik angewendet (Wilde und Hess 2007). Die erste Forschungsphase umfasste eine qualitative Erhebung, gefolgt von einer zweiten Forschungsphase in Form einer quantitativen Querschnittsanalyse. Die Erkenntnisse aus der qualitativen Studie wurden im Design der darauffolgenden Studie berücksichtigt. Die Kombination aus den Erkenntnissen der beiden Studien bildete die Grundlage für die Ableitung der Erkenntnisse für das Design von Informationssystemen zur Unterstützung von Entscheidungen.

\subsection{Erste Forschungsphase (qualitativ)}

Die erste Forschungsphase umfasste eine qualitative Querschnittsanalyse. Es wurden zehn EntscheiderInnen in C-Level-Positionen (CEO, CIO, ...) interviewt (Tab. 1). Die durchschnittlich 75-minütigen Interviews wurden transkribiert und mittels qualitativer Inhaltsanalyse (Mayring 2015) induktiv ausgewertet.

Die so ermittelten Faktoren stammen aus dem sozio-individuellen Bereich der EntscheiderInnen (Erfahrung, Stimmung, Lernen, Selbstbewusstsein, Empfehlung anderer). Aber auch kontext- und entscheidungsimmanente Faktoren, wie die betriebliche Funktion (z. B. Personalmanagement, Marketing, Finanzen), die Bedeutung der Entscheidung (Risiko, Tragweite) und Ressourcen (Kosten, Zeit, vorhandene Daten), wurden identifiziert. Die Faktoren im sozio-individuellen Bereich lassen nicht klar erkennen, welchen Einfluss sie auf das Entscheidungsverhalten haben. Im Gegensatz dazu konnte bei den kontext- und entscheidungsimmanenten Faktoren deren Einfluss festgestellt werden. Hinsichtlich der betrieblichen Funktion, der die Entscheidung zugeordnet werden kann, werden Entscheidungen im Bereich Marketing, Verkauf (Sales) und Personalmanagement (HR) - ,weiche“, schwieriger zu quantifizierende betriebliche Funktionen - eher intuitiv getroffen. Im Gegensatz dazu werden Entscheidungen im Bereich Finanzen - „harte“ faktenorientierte und quantifizierbare betriebliche Funktionen - meist rational getroffen. Auch die Be-

Tab. 1 Beschreibung der InterviewpartnerInnen (MA MitarbeiterInnen)

\begin{tabular}{llll}
\hline ID InterviewpartnerIn & Sektor & Größe (MA) & Position \\
\hline I1 & Maschinen & 220.000 & ManagerIn \\
I2 & Bildung & 2500 & Direktor \\
I3 & IT & 140 & CEO \\
I4 & Realitäten & 500 & CEO \\
I5 & IT & 5800 & Key Account ManagerIn \\
I6 & Finanzen & 1300 & CEO \\
I7 & Consulting & 140 & CEO \\
I8 & Consulting & 20 & CEO \\
I9 & Produktion & 1100 & CEO \\
I10 & Kultur & 400 & CEO \\
\hline
\end{tabular}




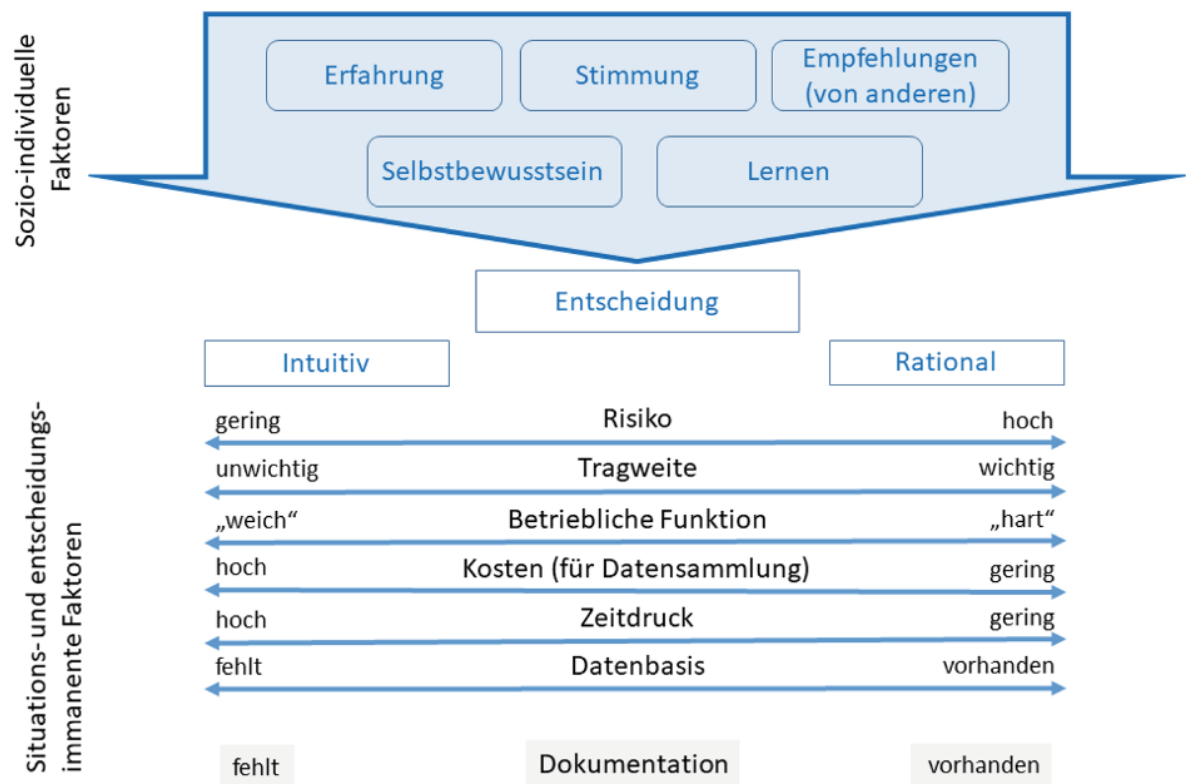

Abb. 1 Sozio-individuelle und situations- und entscheidungsimmanente Faktoren

deutung, also die Tragweite und das damit verbundene Risiko, haben einen Einfluss darauf, wie Entscheidungen getroffen werden. Rational werden vor allem jene Entscheidungen getroffen, die bedeutsam und riskant sind, während eher „unwichtige“ Entscheidungen mit geringem Risiko für das Unternehmen verstärkt intuitiv getroffen werden. Interessanterweise geben die Interviewten an, dass man sich auch mit rational getroffenen Entscheidungen „wohlfühlen muss“, wie zum Beispiel die InterviewpartnerInnen I3 und I4 wortwörtlich formulierten. Die Hälfte gab an, dass sie auch rationale Entscheidungen verworfen haben, wenn diese ihrer Intuition widersprachen und sie ausreichend Selbstbewusstsein entwickelt hatten. Aber auch intuitiv getroffene Entscheidungen wurden versucht im Nachhinein mit Hilfe von Daten zu rechtfertigen. Generell wurde Intuition ausdrücklich als wichtige Fähigkeit für ManagerInnen betrachtet. Auch die Dokumentation von Entscheidungen wurde thematisiert, um diese für das Lernen und zukünftige Entscheidungen nutzen zu können. Dokumentation bietet demnach die Möglichkeit, retrospektiv feststellen zu können, „ob die Entscheidung richtig war", wie InterviewpartnerIn I5 wortwörtlich konstatierte. Die Ergebnisse der qualitativen Interviews sind in Abb. 1 zusammengefasst. Die qualitativen Interviews zeigen aber auch, dass das Treffen von Entscheidungen als eine wichtige Aufgabe des Managements gesehen wird und es sei „oft besser eine schlechte Entscheidung zu treffen, als gar keine“ (Zitat InterviewpartnerIn I7). 


\subsection{Zweite Forschungsphase (quantitativ)}

Auf die explorative Studie folgte eine quantitative Querschnittsanalyse in Form einer Onlineumfrage. 200 EntscheiderInnen wurden dazu eingeladen, 91 davon nahmen daran teil. Der Fragebogen umfasste 17 Fragen, fünf davon zur Demographie der TeilnehmerInnen. Die Ergebnisse sind in Abb. 2 dargestellt.

Im Fragebogen wurde inhaltlich vor allem auf Faktoren eingegangen, die für die Anforderungen an ein Informationssystem von Bedeutung sind. Der Fragebogen wurde basierend auf den Erkenntnissen aus der ersten Forschungsphase erstellt und in zwei Pretests getestet. Die inhaltlichen Fragen betrafen das Bewusstsein über intuitive Entscheidungen (F1), die Identifizier- und Visualisierbarkeit (F2), die Häufigkeit von intuitiven Entscheidungen (F3) sowie die Wahrscheinlichkeit, rationale Entscheidungen aufgrund von Intuition zu verwerfen (F4). Danach wurden Fragen zur Relevanz der Dokumentation von Entscheidungen (F5), der Dokumentation von Entscheidungen im Unternehmen generell (F6) und mit Hilfe von entsprechenden Vorlagen (F7) gestellt. Im Anschluss daran wurden in Form einer offenen Frage die Gründe für das Fehlen von Vorlagen (F8) erhoben. Die restlichen Fragen betrafen Lernen bzw. Erfahrung (F9), empfundene Qualität (F10) und Akzeptanz von Bauchentscheidungen (F11) sowie Einfluss der Intuition auf Entscheidungen (F12). Zu jeder Frage war weiterführende Information vorhanden, die im Online-Befragungstool abgerufen werden konnte (z.B. wurde erklärt, was unter einer wesentlichen Entscheidung zu verstehen ist). Die inhaltlichen Fragen sind im Anhang dargestellt, aus Platzgründen wurden die zusätzlichen Informationen nicht abgebildet. Die Daten wurden mittels deskriptiver Statistik ausgewertet (Abb. 3).

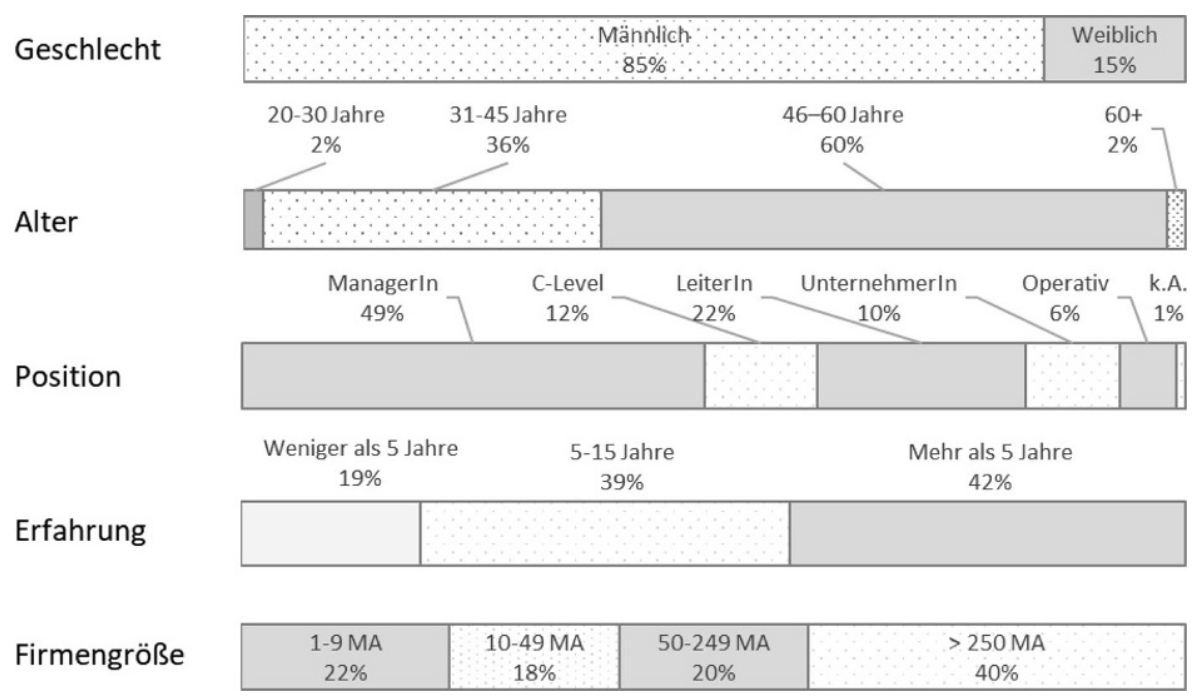

Abb. 2 Demographische Auswertung (MA MitarbeiterInnen) 


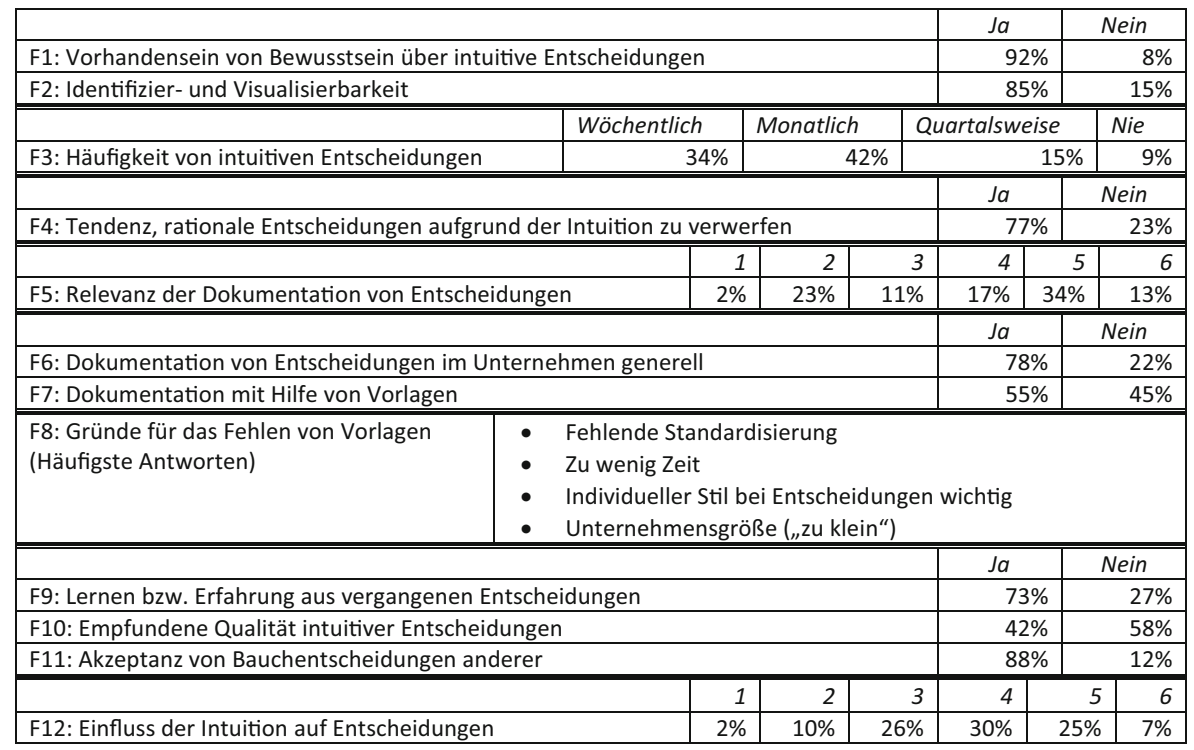

Abb. 3 Deskriptive Auswertung der Umfrage

\section{Erkenntnisse für Informationssysteme}

Aus den Ergebnissen wurden Erkenntnisse abgeleitet, die als abstrakte Anforderungen an ein zu entwickelndes Informationssystem zur Unterstützung der EntscheiderInnen im Umgang mit intuitiven Entscheidungen interpretiert werden können. Zur Strukturierung der Faktoren wird das Mensch/Aufgabe/Technik-System (MAT-System) verwendet, das ein Informationssystem als soziotechnisches System (Anwendungssystem) interpretiert und damit das zentrale Erkenntnisobjekt der Wirtschaftsinformatik beschreibt (Heinrich et al. 2011). Die Mensch-Komponente repräsentiert die Individuen in einer Entscheidungssituation, die mit dem Informationssystem interagieren und auf deren Bedürfnisse es zugeschnitten sein muss. Um der wechselseitigen Beziehung Rechnung zu tragen, ist es essentiell, die organisatorischen (strategischen und administrativen) Rahmenbedingungen zu berücksichtigen, die für diese Rolle relevant sind. Faktoren wie Erfahrung, Erfahrungszuwachs oder persönliches Risikoverhalten sind zu berücksichtigen (Styhre 2011). Die Aufgaben-Komponente ist in erster Linie als „Entscheidungsunterstützung“ definiert. Dies greift aber zu kurz, da Entscheidungen abhängig von der zeitlichen und innerorganisatorischen Reichweite, der Art der Entscheidung (z. B. Personal- oder Investitionsentscheidung) oder dem Entscheidungskontext (z. B. Zeitdruck) sind. Die TechnikKomponente repräsentiert sowohl Technik (hier synonym mit Technologie) im Sinne einer Sachtechnik (z.B. Applikation auf einem Smartphone), als auch die darin abzubildende Entscheidungstechnik (Heinrich et al. 2014). Das Erklärungsmodell des MAT-Systems dient als Grundlage für die Präsentation der Faktoren, die abstrakte Anforderungen an ein Informationssystem zur Unterstützung der intuitiven Entscheidung sind (siehe Abb. 4). Die Faktoren sind weder trennscharf, noch von- 
Abb. 4 Faktoren im MAT-System

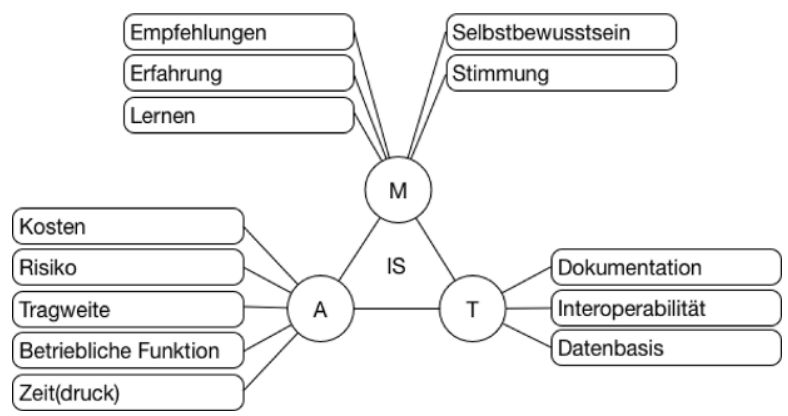

einander unabhängig. Bestehende Korrelationen oder kausale Beziehungen werden nicht berücksichtigt.

\subsection{Mensch-Komponente (M)}

Diese Komponente repräsentiert die EntscheiderInnen mit deren individuellen Persönlichkeiten. Demgemäß ist den folgenden Faktoren Rechnung zu tragen:

- Empfehlungen: Intuitive Entscheidungen sowie die Entscheidung für die intuitive Entscheidung hängen häufig mit der Empfehlung von anderen zusammen. Entsprechend soll das Informationssystem die Möglichkeit bieten, Empfehlungen (für oder gegen eine intuitive Entscheidung) in einer ähnlichen Entscheidungssituation darzustellen. Darüber hinaus soll dokumentiert werden, welche Entscheidungen basierend auf einer Empfehlung getroffen wurden.

- Erfahrung: Einerseits beeinflusst die Erfahrung die Neigung, intuitive Entscheidungen zu treffen. Andererseits wirkt sich Erfahrung auch auf die Fähigkeit aus, die Auswirkungen einer spezifischen intuitiven Entscheidung abschätzen zu können. Daher soll das Informationssystem Erfahrung abbilden können. So kann beispielsweise auf Basis eines persönlichen Profils gezeigt werden, mit welchen Entscheidungen bereits welche Erfahrung gesammelt wurden.

- Lernen: EntscheiderInnen wollen sich weiterentwickeln, deshalb soll das Informationssystem Mechanismen enthalten, die den Lernprozess unterstützen, also z. B. Unterschiede zu getroffenen Entscheidungen in ähnlichen Entscheidungssituationen aufzuzeigen. Zudem kann das Informationssystem (ex-post) zu einer aktiven Auseinandersetzung mit der Entscheidung anregen, indem es voneinander abweichende Entscheidungen in ähnlichen Situationen aufzeigt. Dies bedingt, dass Entscheidungen entsprechend gespeichert und klassifiziert werden. Eine Möglichkeit dazu wäre ein Vektorraum-Modell, in dem Aspekte wie Kosten, Zeitdruck o. ä. als Dimensionen abgebildet werden. Damit kann durch Methoden des Case-Based Reasoning beispielsweise die Ähnlichkeit von Situationen konzeptionalisiert werden.

- Selbstbewusstsein: Da die intuitive Entscheidung (bewusst) gegen die Faktenlage laufen kann, spielt der Faktor Selbstbewusstsein eine wichtige Rolle. Wenn in der Entscheidungssituation die Bauchentscheidung (entgegen der faktenbasierten 
Entscheidung) ein positives Ergebnis erzielt, so kann dies EntscheiderInnen langfristig (positiv) beeinflussen. Daher soll das Informationssystem dies abbilden, um über dieses Feedback die EntscheiderInnen zu bestärken.

- Stimmung (und Tagesverfassung): haben einen Einfluss auf Entscheidungen, die von Menschen getroffen werden. Entsprechend soll das Informationssystem Mechanismen vorsehen, die ungewöhnliches Verhalten konträr zu vergangenen ähnlichen Entscheidungssituationen erkennen können (z.B. ungewöhnlich schnelles Ablehnen einer rationalen Entscheidung). Das Informationssystem könnte einen Hinweis (z. B. Warnmeldung) ausgeben.

\subsection{Aufgabe-Komponente (A)}

Die Komponente repräsentiert die Entscheidung als betriebswirtschaftliche (Management-)Aufgabe. Entsprechend sind die folgenden Faktoren zu berücksichtigen:

- Kosten: können entstehen, wenn eine Entscheidung (rational) nicht getroffen werden kann (z. B. aufgrund mangelnder Datenbasis). Da in der betriebswirtschaftlichen Praxis häufig davon ausgegangen wird, dass eine „schlechte“ Entscheidung besser ist als keine, soll das Informationssystem Möglichkeiten enthalten, auf eine intuitive Entscheidung als Alternative hinzuweisen (z. B. in Form einer Warnmeldung).

- Risiko (der Entscheidung): ist ein wichtiger Faktor für oder gegen eine intuitive Entscheidung. Das Informationssystem soll diesem Umstand Rechnung tragen und das Risiko der Entscheidung entsprechend aufzeigen und visualisieren (z.B. Risiko-Thermometer).

- Tragweite: umfasst die Auswirkungen der Entscheidung für das Unternehmen. Während administrative Entscheidungen eher intuitiv entschieden werden, sind strategische Themen stark mit faktenbasierten Entscheidungen gekoppelt. Das Informationssystem muss daher diesen Zusammenhang, analog zur Komponente Risiko, abbilden.

- Betriebliche Funktion: Entscheidungen im Rahmen bestimmter betrieblicher Funktionen (z. B. Personal) werden häufiger intuitiv getroffen. Dies soll im Informationssystem dargestellt sein (z.B. eine Matrix, die visualisiert, wie Entscheidungen im Kontext betrieblicher Funktionen getroffen wurden).

- Zeit(druck): ist analog zum Faktor Kosten zu sehen. Kann die Grundlage für eine faktenbasierte Entscheidung nicht zeitnah geschaffen werden, so ist die intuitive Entscheidung als Alternative aufzuzeigen (siehe Kosten).

\subsection{Technik-Komponente (T)}

Die Komponente repräsentiert die (informations)technischen Einflüsse auf die intuitive Entscheidung, die sich nicht direkt aus der Umsetzung der Anforderungen aus Mensch- und Aufgaben-Komponente ableiten: 
- Dokumentation: ist eine Querschnittsfunktion, die als Voraussetzung für die Umsetzung einiger Faktoren (z.B. Empfehlung, Lernen oder Tragweite) notwendig ist.

- Interoperabilität: Das Informationssystem soll in hohem Maße interoperabel mit existierenden Entscheidungsunterstützungssystemen im Unternehmen sein. Dazu gehören Schnittstellen zu ERP- und/oder BI-Systemen, um wechselseitige Beziehungen von Entscheidungen abzubilden.

- Datenbasis: Intuitive Entscheidungen werden auch deshalb getroffen, weil mangels Datenbasis keine faktenbasierte Entscheidung getroffen werden kann. Entsprechend soll das Informationssystem diese Situation hinreichend abbilden können, also aufzeigen können, auf welche Daten für die jeweilige Entscheidung zurückgegriffen werden kann. Insofern besteht hier ein Anknüpfungspunkt zu klassischen Entscheidungsunterstützungssystemen. Diese Komponente ist auch eng mit den Faktoren Kosten und Zeit verknüpft.

\section{Schlussfolgerungen, Einschränkungen und weitere Forschung}

Intuitive Entscheidungsfindung ist trotz eines ausgerufenen Zeitalters von Big Data und automatisierten Entscheidungen eine Realität für ManagerInnen. Das Ziel der Arbeit war es, Anforderungen an zukünftige Informationssysteme abzuleiten, die Individuen unterstützen sollen, sich bewusst für oder gegen eine intuitive Entscheidung zu entscheiden, Entscheidungen zu beurteilen und aus diesen Entscheidungssituationen für zukünftige Entscheidungen zu lernen. Diese wurden mit Hilfe einer zweistufigen empirischen Untersuchung erhoben. Es wurden fünf sozio-individuelle Faktoren (Erfahrung, Stimmung, Empfehlung von anderen, Selbstbewusstsein, Lernen), sechs situations- und entscheidungsimmanente Faktoren (Risiko, Tragweite, betriebliche Funktion, Kosten, Zeitdruck, Datenbasis) sowie die Dokumentation von Entscheidungen als Basis in den qualitativen Interviews identifiziert und mit Hilfe der quantitativen Studie validiert und vertieft. Die Erkenntnisse dienten danach als Grundlage für die Ableitung der Anforderungen für das Design eines Informationssystems zur Unterstützung von EntscheiderInnen im Umgang mit intuitiven Entscheidungen. Faktoren wie Empfehlungen, Erfahrungen und Lernen der EntscheiderInnen, aber ebenso Entscheidungskontext inklusive Kosten und Risiko sollten dementsprechend in einem Informationssystem abgebildet werden. Insgesamt wird damit EntscheiderInnen ein besseres Verständnis von Entscheidungssituationen verschafft und eine Rückkopplungsschleife bereitgestellt, die es ermöglicht, aus früheren Entscheidungen zu lernen.

Der mögliche Einsatz eines solchen Informationssystems ist daher in zweifacher Hinsicht zu sehen: (1) Es kann in Organisationen genutzt werden, um einzelne EntscheiderInnen zu unterstützen. Darüber hinaus würde es der Organisation auch ermöglichen, Entscheidungsstile und -ergebnisse zu bewerten und darauf aufbauend Prozesse und Strukturen anzupassen, um Entscheidungstypen und -methoden zu optimieren. (2) Es ermöglicht der Forschung Einblicke in reale Entscheidungen, ihre Kontexte und ihre Ergebnisse sowie etwaige Verhaltensänderungen durch das bereit- 
gestellte Feedback. Dies kann dazu beitragen, die Informationssystemunterstützung weiter zu verbessern, aber auch menschliche Entscheidungsprozesse besser zu verstehen.

Dieser Beitrag ist als Grundlage für weitere Forschungsarbeiten im Bereich der intuitiven Entscheidungsfindung zu sehen. Es wurde ein Grundstein für die Entwicklung eines entsprechenden Informationssystems gelegt, welches sowohl als Artefakt im Sinne eines konstruktionsorientierten Ansatzes besteht, aber auch als Erhebungsinstrument zur Sammlung empirischer Daten eingesetzt werden kann. Es wurde daher ein korrespondierender Prototyp des oben beschriebenen Informationssystems entwickelt, der derzeit in realen Entscheidungssituationen getestet wird.

Funding Open access funding provided by Johannes Kepler University Linz.

\section{Anhang}

\section{Fragebogen (inhaltliche Fragen)}

- F1: Haben Sie schon einmal bewusst zwischen Bauchentscheidung und rationaler Entscheidung unterschieden? (Skala: J/N)

- F2: Ist es aus Ihrer Sicht möglich, Entscheidungen zu erkennen und für andere sichtbar zu machen? (Skala: J/N)

- F3: Wie oft kommt es vor, dass Sie wesentliche Unternehmensentscheidungen aus dem Bauch heraus treffen? (Skala: wöchentlich/monatlich/quartalsweise/nie)

- F4: Haben Sie schon einmal eine Bauchentscheidung getroffen, obwohl die rationalen Fakten dagegensprachen? (Skala: J/N)

- F5: Auf einer Skala von 1-6, wieviel Wert legen Sie auf die Dokumentation von getroffenen Entscheidungen? (Skala: 1: keinen Wert - 6: sehr viel Wert)

- F6: Werden in Ihrem Unternehmen Entscheidungen dokumentiert? (Skala: J/N)

- F7: Wenn Ja, gibt es dafür Vorlagen? (Skala: J/N)

- F8: Wenn Nein, warum nicht? (Offene Frage - Anmerkung: bezieht sich auf F7)

- F9: Falls Sie eine Entscheidung zu treffen haben, und diese (oder eine ähnliche) Entscheidung wurde in der Vergangenheit aus dem Bauch oder rational entschieden, werden Sie diese Entscheidung in derselben Art wieder treffen? (Skala: J/N)

- F10: Glauben Sie, dass rationale Entscheidungen die gleiche Qualität wie Bauchentscheidungen aufweisen? (Skala: J/N)

- F11: Akzeptieren Sie Bauchentscheidungen, die von anderen Personen getroffen werden? (Skala: J/N)

- F12: Auf einer Skala von 1-6, wie sehr denken Sie fließt bei einer Entscheidung Ihr Bauchgefühl mit ein? (Skala: 1: wenig - 6: sehr)

Open Access Dieser Artikel wird unter der Creative Commons Namensnennung 4.0 International Lizenz (http://creativecommons.org/licenses/by/4.0/deed.de) veröffentlicht, welche die Nutzung, Vervielfältigung, Bearbeitung, Verbreitung und Wiedergabe in jeglichem Medium und Format erlaubt, sofern Sie den/die ursprünglichen Autor(en) und die Quelle ordnungsgemäß nennen, einen Link zur Creative Commons Lizenz beifügen und angeben, ob Änderungen vorgenommen wurden. 


\section{Literatur}

Agor WH (1986) The logic of intuition: how top executives make important decisions. Organ Dyn 14:5-18

Alavi M, Leidner DE (2001) Knowledge management and knowledge management systems: conceptual foundations and research issues. MIS Q 25:107-136

Dane E, Pratt MG (2007) Exploring intuition and its role in managerial decision making. Acad Manage Rev 32:33-54

Day RH (1971) Rational choice and economic behavior. Theory Decis 1:229-251

Epstein S (1994) Integration of the cognitive and the psychodynamic unconscious. Am Psychol 49:709-724

Gintis H (2000) Beyond homo economicus: evidence from experimental economics. Ecol Econ 35:311-322

Goll I, Rasheed AM (1997) Rational decision-making and firm performance: the moderating role of environment. Strateg Manage J 18(7):583-591

Heinrich LJ, Heinzl A, Riedl R (2011) Wirtschaftsinformatik: Einführung und Grundlegung, 4. Aufl. Springer, Berlin

Heinrich LJ, Riedl R, Stelzer D, Sikora H (2014) Informationsmanagement: Grundlagen, Aufgaben, Methoden, 11. Aufl. De Gruyter Oldenbourg, München

van Helden A, Buck-Emden R, Alda S (2018) Präskriptive Entscheidungsunterstützung für wissensintensive Geschäftsprozesse. HMD Prax Wirtschaftsinform 55:197-222

Huang L (2017) The role of investor gut feel in managing complexity and extreme risk. Acad Manage J. https://doi.org/10.5465/amj.2016.1009

Issack TS (1978) Intuition: an ignored dimension of management. Acad Manage Rev 3:917-922

Kahneman D, Frederick S (2002) Representativeness revisited: attribute substitution in intuitive judgment. In: Gilovich T, Griffin D, Kahneman D (Hrsg) Heuristics and biases. Cambridge University Press, Cambridge, S 49-81

Lank AG, Lank EA (1995) Legitimizing the gut feel: the role of intuition in business. J Manage Psychol 10:18-23

Mayring P (2015) Qualitative Inhaltsanalyse: Grundlagen und Techniken, 12. Aufl. Beltz, Basel

Mertens P (1999) Geschichte und ausgewählte Gegenwartsprobleme der Wirtschaftsinformatik. In: 100 Jahre Betriebswirtschaftslehre in Deutschland, 1. Aufl. Vahlen, München, S 183-195

Meyer R (2000) Entscheidungstheorie, 2. Aufl. Gabler, Wiesbaden

Milkman KL, Chugh D, Bazerman MH (2009) How can decision making be improved? Perspect Psychol Sci 4:379-383

Pfeffer J, Sutton RI (2006) Evidence-based management. Harv Bus Rev 84:1-62

Sadler-Smith E, Shefy E (2004) The intuitive executive: understanding and applying 'gut feel' in decisionmaking. Acad Manag Exec 18:76-91

Savage LJ (1951) The theory of statistical decision. J Am Stat Assoc 46:55

Sayegh L, Anthony WP, Perrewé PL (2004) Managerial decision-making under crisis: the role of emotion in an intuitive decision process. Hum Resour Manage Rev 14:179-199

Shapiro S, Krishnan HS (2001) Memory-based measures for assessing advertising effects: a comparison of explicit and implicit memory effects. J Advert 30:1-13

Simon HA (1987) Making management decisions: the role of intuition and emotion. Acad Manag Exec 1:57-64

Sitkin SB, Weingart LR (1995) Determinants of risky decision-making behavior: a test of the mediating role of risk perceptions and propensity. Acad Manage J 38:1573-1592

Stanovich KE, West RF (2000) Individual differences in reasoning: implications for the rationality debate? Behav Brain Sci 23:645-665

Styhre A (2011) Practice and intuitive thinking: the situated nature of practical work. Int J Organ Anal 19:109-126

Wilde T, Hess T (2007) Forschungsmethoden der Wirtschaftsinformatik: Eine empirische Untersuchung. Wirtsch Inform 49:280-287 